\title{
Designing a Touch Screen Communication Device to Support Social Interaction amongst Older Adults
}

\author{
Julie Doyle \\ School of Public Health, \\ University College Dublin \\ julie.doyle@ucd.ie
}

\author{
Zoran Skrba \\ TRIL Centre \\ University College Dublin \\ zoran.skrba@trilcentre.org
}

\author{
Ronan McDonnell \\ Centre for Health Informatics \\ Trinity College, Dublin, Ireland \\ mcdonnra@scss.tcd.ie
}

\author{
Ben Arent \\ TRIL Centre \\ University College Dublin \\ ben.arent@trilcentre.org
}

\begin{abstract}
As people age, social connections can be lost due to a number of factors. Technology can enhance an older person's social connectedness, facilitating the creation of new connections, as well as the maintenance of existing ones. As part of the Building Bridges project, a communication device was deployed in 9 older adult's homes and evaluated over a period of 7-9 weeks. The goals of the study were to assess the usability of the device, to explore attitudes towards it and to gather insights into potential target user groups who may benefit from such technology. We present our findings which highlight the importance of feedback and confirmation in increasing the usability of a technology device for older adults. Emergent themes surrounding older adults' attitudes to using such technology to keep in touch, include the importance of perceived usefulness and the ability to have some level of control over when communication occurs and with whom.
\end{abstract}

Older adults, social connectedness, communication technology, usability, usage, perceived usefulness

\section{INTRODUCTION}

As people age, social connections can be lost as a result of widowhood, dispersed family members or a shrinking peer network. Further inhibitors to reduced social interaction include illness, a lack of mobility or a fear of falling, each of which can confine a person to their home, reducing their engagement in social activities and potentially resulting in a loss of independence. Such events can have an adverse effect on a person's health. The health risks associated with lower levels of social integration have been much discussed in the literature and are comparable in magnitude to the risks associated with cigarette smoking, high blood pressure and obesity [1]. As such, fostering social communication among older adults is vital in maintaining a healthy, independent life. Technology can facilitate communication between older adults and their peers and family members, stimulating new relationships and maintaining existing ones [3]. However, technology can often represent a barrier for older adults, acting as an inhibitor to usage rather than a facilitator. There are many reasons for this including unfamiliarity, computer anxiety and inaccessible technology [2]. Furthermore, cognitive disabilities resulting from age degenerative processes can significantly increase the learning curve of older adults, making it more difficult and time consuming for this group to learn new skills, compared to younger adults. Physical impairments related to sensory loss are another obvious effect of ageing [9]. Such impairments affect visual, auditory and tactile capabilities, further distancing older adults from technology. Sainz Salces et al [16] provide a detailed discussion on the physical and cognitive effects of ageing. The above mentioned factors are not only important in designing a usable system for older adults. They also affect whether or not an older adult might want to use such technology. While it is generally agreed that older people are capable of learning new skills [7], as noted in [15] the effort required to learn something new may be perceived as not worth the trouble for the expected gain. Therefore, it is important to understand what might motivate older people to want to use such technology. Designers of applications that target older adults as a user group must understand this cohort's attitudes towards technology and communication and ensure applications are designed with their unique needs in mind. In this paper we discuss the design and evaluation of a touch screen communication device, Building Bridges, that enables older adults to communicate with their peers. We decided on a touch screen as such devices remove the need for peripheral, often unfamiliar input devices, such as a mouse or keyboard, and therefore have great potential to be accessible to older adults [11]. Furthermore, a stand alone device has benefits in that it allows us to hide much of the complexity of a computer. We present details of a pilot home-based deployment of the Building Bridges device, which took place over a period of 7-9 weeks with 9 older 
adults. The aim of this study was to examine usage and usability of the device, as well as to gather user opinion on the device and its concept. While this initial trial connected strangers, we were also interested in whom else older adults might be interested in using the device to communicate with. Our findings have implications on the design of communication technologies in terms of both ensuring usability and what groups of older adults may benefit from it.

\section{BUILDING BRIDGES FOR SOCIAL CONNECTION}

The aim of the Building Bridges project is to use communication technology to foster social connectedness among older adults, their peers and family and friends, reducing the risk of loneliness and social isolation in this cohort. To this end, we have developed a device for the home that provides older adults with the opportunity to connect with their peers, family and friends through the shared experience of a video or radio broadcast (such as documentaries, news, health bulletins). Following each broadcast, listeners have the option to take part in a group chat. Further functionality includes individual or group calls, a (textual) messaging service, and most recently a 'tea room' which represents a chat forum. The current device can be seen in Figure 1. With regard to communication technologies, the exposure of older adults to new, lightweight methods of communication differs vastly to that of younger adults. As noted in [14],"the idea of updating a twitter feed may be as alien to many older people as the possibility of sending a telegram is to a teenager". However, many studies have illustrated the receptiveness of older adults to using e-mail as a form of communication and that such communication is effective in increasing social connectedness among this cohort [3], [14]. Czaja et al [2] conducted a series of focus groups with older adults during which they found that benefit was considered one of the most important reasons for communication in a specific situation. Forms of communication considered were telephone, email, a home visit and a mobile phone.

The Internet and computer usage have been evaluated for effectiveness in reducing social isolation. Findlay reported that internet usage, especially 'senior-friendly' websites seemed to alleviate feelings of social isolation and loneliness [6]. White et al reported that in older people in a mix of retirement and sheltered housing sites that received group training in computer use, followed by 5 months of access to computers, there was a trend towards more confidence than a control group, as well as less loneliness and depression [19]. More recently, social t.v. has been investigated as a means of connecting older adults [17]. As such, technology interventions represent a promising means of enabling social connection.

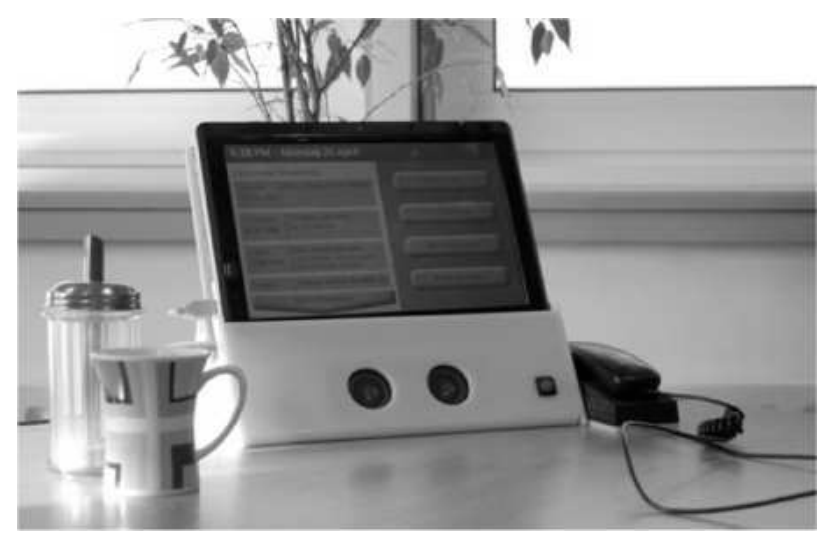

Figure 1: The Building Bridges Device

The Building Bridges user-centred design process consisted of 4 phases and involved a multidisciplinary team, including ethnographers, social scientists, clinicians, interaction designers, computer scientists and engineers. Phase 1 involved ethnographers and social scientists visiting a number of older adults in their homes to conduct expert interviews, helping us to understand and define the dynamics of the problem, as well as providing us with an insight into the attitudes of older adults towards technology and communication. Phase 2 consisted of developing the concept. The ethnographic data was analysed to identify themes for technological opportunities. We then conducted a series of six focus groups, each with 5-20 participants, whereby storyboards were used to explain the concept of the research, as opposed to the technology that would be involved. We took this approach as older people are typically unaware of the possibilities of new technologies, which can limit their ability to contribute actively to a discussion on technological requirements [21]. This facilitated an open discussion among participants, the key points of which guided us in the design of a unified technological concept. Following this phase, the key functions the device should have were identified. These included a broadcast feature to provide a shared experience to act as a topic of conversation, the ability to make calls and to send messages. Phases 1 and 2 have been described in more detail elsewhere [18]. Having established the core features of the device, Phase 3 involved identifying the technical requirements for hardware and software and the development of a prototype. In terms of hardware, it was decided to develop the system on a touch screen device. Given that a touch screen acts as both the method of user input and system output and removes the need for a mouse and keyboard, it is ideally suited to older adults, being easier to learn [11]. Furthermore, it has been shown that a touch-based user interface can be successfully adopted by older users regardless of their physical or cognitive weaknesses [8]. The 
software application was designed with an Adobe Flash front end and a C\# backend. We decided to use VolP technology and chose Skype infrastructure as it supported speedy development and deployment of the system backend. Thus we could concentrate on the UI development. C\# interfaces with the Skype client for all call control and message events. Designing the Building Bridges interface was a critical part of the design process. Existing guidelines for designing accessible interfaces for older adults provided a useful starting point for our initial design [20], [10], [5]. Considerations included vision (Arial font greater than 14-point, high colour contrast to the background), dexterity (ensure buttons are large and no double clicks are required), attention (do not use pop-up windows, moving text or animations), cognition (limit the functionality and number of buttons per screen, avoid dropdown menus, use bold navigation tools), computer knowledge (avoid technical terminology) and button affordance (ensure buttons are easily recognised by giving them a 3-D appearance, have a consistent representation throughout the system and avoid placing non-functional text in boxes to ensure they do not appear as a button). In an effort to identify any additional design requirements for our touch screen system, the initial prototype was evaluated as part of a one-off session in 6 participants homes using a think-aloud protocol. This first prototype presented for evaluation can be seen in Figure 2 (left most image). It consisted of tabbed menus for moving through features and a handwriting feature for composing messages. Based on feedback from users, it was decided to avoid tabbed menus as they caused confusion for older adults. As such, it was decided to make features accessible through buttons on a 'Main Menu' screen (Figure 2 -centre image). Furthermore, participants expressed concern about how their handwriting would appear to other participants "My handwriting is a lot better than that on paper... that looks like it's been written by a four-year old". This is in contrast to other studies, whereby handwritten communication was considered a more personal form of communication, similar to letter writing [14]. However, this is likely because the studies mentioned concerned communication among family and friends, whereas within Building Bridges participants would also be interacting with people initially unknown to them. We therefore decided to implement a touch-based keyboard for composing messages (Figure 2 -centre image). The centre image of Figure 2 represents the design that was evaluated in our first home deployment discussed below in this paper, while the right most image of Figure 2 represents the refined design being evaluated in our currently ongoing home deployment.

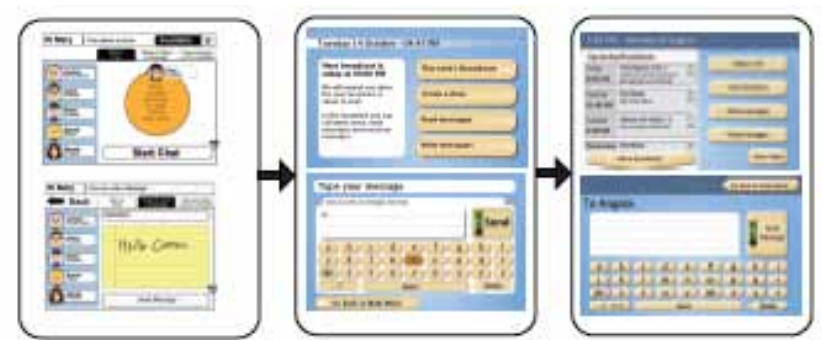

Figure 2: Iterations of the Building Bridges Interface Design

Whilst focus groups and once-off evaluation sessions are important for initial prototype design and development, to gain insightful data regarding the usage, usability and impact of technologies on older adults in real settings, home deployments are necessary. Many usability problems only emerge after usage over time in real contexts. Furthermore, participants will have more realistic opinions and perceptions of the device if given the opportunity to use it over a period of time, rather than in a once-off evaluation session. As such, Phase 4 of our design process involved deploying the Building Bridges device in older adults' homes over a number of weeks. This evaluation, and our findings are the focus of the remainder of the paper.

\section{HOME DEPLOYMENT EVALUATION}

A total of ten participants, over the age of 60 , took part in our home deployment evaluation. The participants were divided into two groups of 5 . This decision was made based on the restrictions of setting up a conference call within the Skype API, which limited the number of participants to 5 . A newer version of the API has since been released which provides support for larger numbers. Group 1 consisted of 3 men and 2 women with a mean age of 77.6 (range 6982) and this trial took place over a period of 9 weeks. 3 participants were widowed and living alone, while 2 lived with their spouse. All 5 participants from Group 1 said they had limited computer experience, but would have used the internet previously. Group 2 consisted of 4 women and 1 man with a mean age of 71.4 (range 66-77). This trial lasted 7 weeks (beginning two weeks after Group 1's trial). However, during the trial the male participant from Group 2 was hospitalised, and as such had to withdraw from the study. 3 of Group 2's participants were widows living alone, while 1 lived with a spouse. Of the 4 participants, 3 had little previous computer usage, while 1 used the internet regularly. The deployment of Building Bridges involved a home visit with each participant, during which the device was installed and all functionality was demonstrated and explained. The participant then trained on the device by interacting with it. At this stage a 'dummy' broadcast was set 
up to allow the participant to join and listen. We also asked them to try out the other features. During this training process we used the think-aloud protocol to gauge first opinions, concerns and expectations of the device. The entire home visit lasted about one hour. Following this, participants were left to use the device over a period of 7-9 weeks. Throughout this time we monitored usage through logged interactions. At the end of the trial, we conducted a usability observation session, whereby participants were asked to think-aloud as they performed a number of system tasks. Following the observation session, a post-task interview probed participants for their opinions on the device, their experience over the trial period, how they felt about being contacted by, or making contact with others, as well as whether they felt the device would currently be of benefit to them. This lasted between 60 and 90 minutes. The aim of this particular trial was not to determine categorically whether the device improved a person's social connectedness. A longer, randomised control trial would be necessary to determine this. The main goals were to explore attitudes towards the device, following usage over a number of weeks, to determine whether it was usable for older adults and to gain more insight into potential target user groups (other than socially isolated) who might also benefit from the device.

\subsection{Evaluation Findings -Usage and Usability}

This section highlights our findings from the study, gauged through analysing usage logs in addition to the end of trial observation. 3.1.1 Usage of Features In examining usage logs, we were interested in the total number of broadcasts joined, calls made and messages sent (Table 1). The concept of the system was to use the broadcasts to initially connect people, and once people had met through this medium they could then use the call or messaging feature to further their contact. However, many participants 'broke the ice' through the messaging feature, sending out a group hello or welcome message to all participants in their address book. There was a general feeling from participants that their participation in broadcasts was low as they committed participants to a specific time. We aimed to appeal to a majority with the times of broadcasts, taking into account information gathered in focus groups and by ethnographers on the project regarding the daily activities of older Irish adults. For example, many older Irish adults attend morning mass between 9 and 11. However, it is difficult to find broadcast times to suit all. Our participants each had favourite T.V. or radio programs that they liked to watch, or listen to for example, and sometimes the broadcast would clash with these. Participants suggested we provide an archive of broadcasts so that they can be listened to at a later stage if missed. With regard to broadcast content, participants generally felt that health lectures would be a good feature of the device. The most common suggestion regarding the messaging feature was to provide more space to write messages. The feature aimed to be a short postcard style message, and had space for 160 characters. This lightweight form was considered too short for all participants. The general attitude was that people would have liked to write letter style messages. Also, unlike short-hand communication language used by younger people today, older people do either not know, or not want, to use this. For 7 of the 9 participants, more calls were made than messages sent. Participant 1d, however, preferred to send messages. While he liked to receive calls, he didn't like to make them, for fear that he might disturb someone or they may not want to talk. Communication technologies have huge potential to enhance the lives of socially isolated older adults, if this cohort are willing to use such technology. However, in an uncontrolled home environment, usage can neither be forced nor controlled. Participants were advised to use the device as little or as much as they pleased. In identifying patterns of usage during the trial, usage appears typically to peak mid-trial after which it steadily declines. Post-trial interviews with participants indicate that this may be mainly due to a 'novelty factor' of the technology. As such, we have integrated a number of new features into the current version of the Building Bridges system, the aim of which are to increase the 'on time' of the device and encourage usage. We developed a picture frame feature which displays photos as a screen saver. We are currently conducting a home trial that connects older adults with their family and friends, as well as strangers. The family and friends were asked to email photos to a dedicated email address, which allowed us to display these photos on an individual's device. Other studies have commented on the benefits of such a photo feature in connecting parents and children who are separated [4], whereas the Georgia Tech 2-link device allows residents of a retirement community to share photos in addition to schedules and messages [12]. A second new feature is an always-on 'tea room', which participants may enter at any time for a chat. The tea room shows a live feed from a webcam in Dublin's city centre, while if there is only 1 person in the tea room, music is played.

\subsubsection{Error Rates}

During these observation sessions, we noticed a number of usability issues, in terms of errors made, across participants. A detailed analysis of the log files for each participant over the 7-9 weeks revealed that many of these issues recurred throughout the trial period, as opposed to being once-off issues observed under observation conditions. 


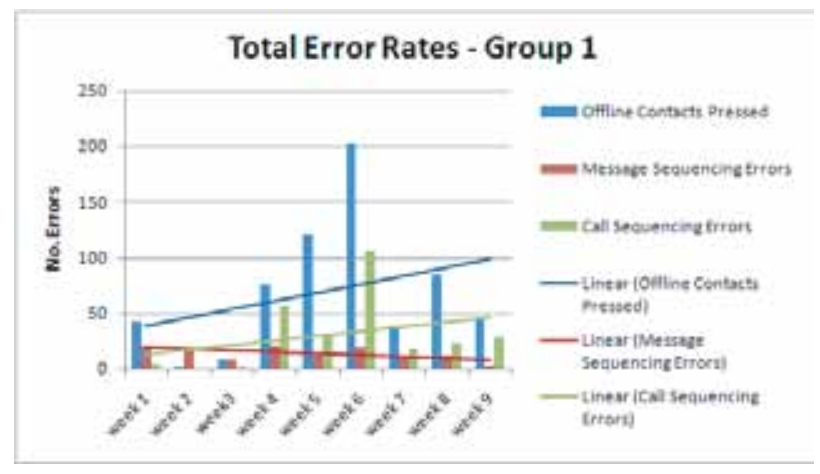

Figure 3: Total Error Rate for Group 1

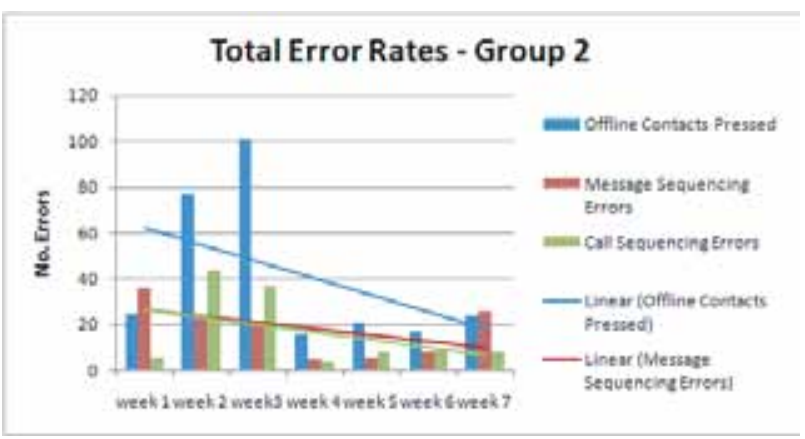

Figure 4: Total Error Rate for Group 2

Sequencing of events in the Call Screen and Message Screen A certain sequence of events is necessary for a participant to call one or more of their contacts (other participants). The participant must first choose who they would like to call from their contacts, which appear on the left of the screen. Once the contact's avatar moves into the Call circle, the 'Call' button becomes active. A line of text on the screen instructed the participant to 'first choose contacts' to call. This sequencing was chosen for its logic in moving from left to right across the screen. However, in our observation session at the end of each home trial, we observed 6 of the 9 participants attempting to press the Call button before having selected participants to call. As such, nothing happened on the screen, as the Call button was only activated if contacts had first been selected. In some cases, participants corrected themselves after a couple of attempts. The majority, however, required input from the evaluator instructing them to 'read the text on the screen'. In examining the sequence of touch events in our logged data over the period of the trial, we noticed this error being made on a number of occasions. Results can be seen in Figure 3 (Group 1) and Figure 4 (Group 2). These errors may have been due to end user mental models around creating a call. Traditionally on a landline phone the user first picks up the phone before dialling the number. As such, further analysis of this task is required to determine the ideal sequencing of events. For our current deployment, we have addressed the issue by providing an error-correction message if the participant tried to press the Call button without first having selected a contact. We also noted the same sequencing errors in the Message Screen, which uses the same model of interaction and requires the same sequencing of events to occur (Figures 3, 4).

\section{Trying to call offine contacts}

Within the Call screen, a differentiation was made between online and offline contacts. Specifically, online contacts were coloured beige, while offline contacts were greyed out. During our observation session, we asked participants how they would normally go about making a call. We noted that often, participants tried to select an offiine contact, whereby they received no indication that this was wrong and often they would attempt to select the offiine contact again. When probed why they thought nothing was happening on the screen, they responded that they did not know, indicating that they hadn't yet learned the difference between online and offline contacts (despite this having been a key point of our training session during deployment). Analysis of the logs over the trial indicates this was a recurring problem (Figures 3, 4). We analysed our data by logging all of the participants' presses on the Call screen. These were filtered by $x, y$ coordinates whereby we compared presses on the participant address book screen to the online status of the pressed contact at

Table 1: Usage Data for Group 1 over 9 Weeks and Group 2 over 7 weeks

\begin{tabular}{|l|l|l|l|l|}
\hline Participant ID & $\begin{array}{l}\text { Total Calls } \\
\text { Made }\end{array}$ & $\begin{array}{l}\text { Total Messages } \\
\text { Sent }\end{array}$ & $\begin{array}{l}\text { Total Broad- } \\
\text { casts } \\
\text { (Out of 29) }\end{array}$ & $\begin{array}{l}\text { Chats Joined } \\
\text { After } \\
\text { cast }\end{array}$ \\
\hline $1 \mathrm{a}$ & 30 & 10 & 10 & 5 \\
\hline $1 \mathrm{~b}$ & 37 & 21 & 13 & 6 \\
\hline $1 \mathrm{c}$ & 33 & 25 & 10 & 4 \\
\hline $1 \mathrm{~d}$ & 6 & 19 & 10 & 2 \\
\hline $1 \mathrm{e}$ & 4 & 9 & 6 & 3 \\
\hline $2 \mathrm{a}$ & 43 & 21 & 10 & 1 \\
\hline $2 \mathrm{~b}$ & 17 & 4 & 6 & 1 \\
\hline $2 \mathrm{c}$ & 30 & 17 & 14 & 2 \\
\hline $2 \mathrm{~d}$ & 21 & 16 & 9 & 1 \\
\hline
\end{tabular}


that time.

\section{Pressing buttons in error - Positioning problems}

On each screen of the Building Bridges device there is a button that allows the user to return to the Main Menu. During this trial, the button was positioned at the bottom left of each screen, to prevent screen coverage. We observed users inadvertently hitting this button with their wrists or knuckles when they were aiming to press another button on the screen. This caused the user to be returned to the Main Menu, when this was not their intention, or within the screen to write a message, a pop-up would ask the user if they would really like to go back. Each time the 'No' button was pressed on the pop-up, we qualified an accidental press on the 'Back to Main Menu' button. The frequency of this problem throughout the trial, within the screen to write a message, can be seen in Figure 5. As illustrated, apart from a small number of participants $(2 b, 2 c, 2 d)$ the average number of times this button was pressed compared to the total number of messages a participant sent, is quite high. Participant 2a, for example, sent a total of 21 messages and accidentally hit the 'Back to Main Menu' button 30 times. We have since moved this button to the top right hand corner of the screen. Initial results from a current trial indicate that this has reduced the number of accidental hits.

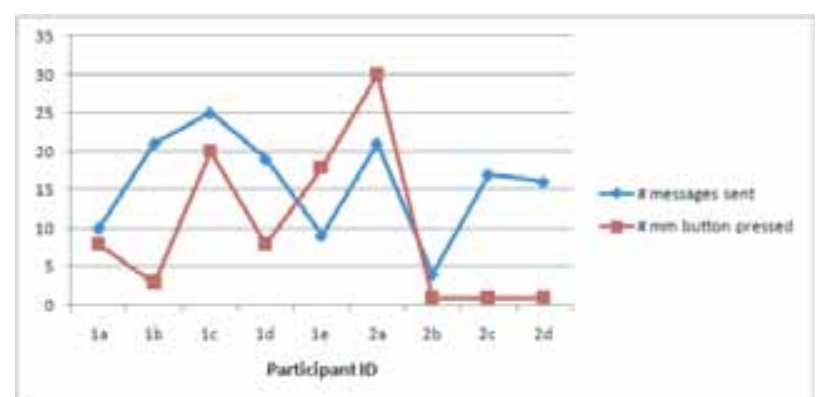

Figure 5: Graph depicting the number of messages sent per participant during Trial 1 versus the number of times the 'Back to Main Menu' button was inadvertently pressed

Ensuring usability is an important factor in encouraging usage. If technology becomes frustrating, people will lack motivation to use it. During our trial we identified a number of usability issues which potentially had negative effects on the participant learning the technology over time. We are aware that many of the errors that were discovered may have been avoided through a $\mathrm{HCl}$-led design. Unfortunately, the $\mathrm{HCl}$ person on the team only came on board just as the trial was beginning. However, we feel it is important to highlight these issues here and to stress the importance of following $\mathrm{HCl}$ guidelines for designing interfaces, particularly where older people are concerned. As indicated in our study, confirmation, feedback of screen actions and prompts for error correction are of the utmost importance, not only to provide participants with a sense of what they are doing is correct, but also to allow them to learn from their errors. Other issues include being careful with the positioning of buttons, avoiding the use of icons on buttons and supporting intuitive input, for example providing a stylus as older adults may suffer from dexterity problems and a stylus can represent a more accurate input device than the finger. One male participant found the device wasn't very responsive: "If it could be made a little more sensitive to the touch, in such a way that people who have a heavy hand can adjust... I obviously haven't learned how to touch it, you know. It's hard to move these things (icons of people)sometimes". However, a participant in the other group found that if she used the tip of a pencil or pen to write messages, the device was a lot more responsive. This participant wrote a message to all other participants in her group to tell them about her 'discovery'. Despite the errors observed, participants generally perceived the device as being easy to use. One participant commented "Now, it'd be very easy to use for someone who knows nothing about computers, I mean a child could use it, bless us you've only to read the screen and press. You couldn't go wrong". There is a general notion that older adults are fearful of technology or unwilling to try it. Within our trial, fear of technology was definitely not an issue. We found participants would be more than willing to try a new technology and to accept it into their home for long term use if they thought it would be of value to them. One benefit of this trial was that it introduced many people to the idea of using some form of technological communication. While one participant felt the device was not for her, she did comment that she had asked her son to get her a laptop so she could begin to use email, saying she had not seen the point of it before using the Building Bridges device.

\subsection{Evaluation Findings -Perceptions and Attitudes}

The goals of the Building Bridges device are to facilitate communication and to fight loneliness by connecting the unconnected and maintaining or augmenting existing connections. In this section, we discuss the findings from our evaluation, focusing on themes that emerged surrounding both the device and communication with others.

\subsubsection{Perceived Usefulness -Who is it for?}

As mentioned in the previous section, a participant's perception of the value of the technology to them, at their current point in life, is a large factor in level of usage. This represents a strong emergent theme as to who might benefit from this system. Although a person's social network may shrink as they age, this 
could be intentional, as the person focuses solely on good relationships, ridding themselves of the bad. As such, it is important to realise that many older adults may be happy with their current social network and may not feel the need for a communication device. Understanding attitudes towards this is important for the design of a system such as Building Bridges. Those participants who didn't use the device very much cited its lack of benefit for them at their current stage in life: "If you were bed-bound, housebound or chair-bound you might use it, if I wasn't as mobile as I am and there were equally knackered(tired or infirm) people available, that they would always be there";"'lt might be useful for some people, but not for me". Itmaybe necessary to define user models in further developing Building Bridges. For example, one such group might be geographically isolated older adults who have similar needs in terms of being confined to their homes because they don't have access to a transport network. Participants who are not socially lonely may benefit from using the device to connect with family members. Furthermore, all participants felt that health lectures would be a useful addition to the device. As such, ARGs might be another user group as they typically run events regularly, but due to various reasons many members can not always physically attend. Functionality on the device could therefore be adapted to suit different user models.

\subsubsection{Attitudes Towards Communication - Calls and Messages}

How participants feel about having a device such as Building Bridges in their home and the ability of people to call them at any time is also important to note. Some participants had specific ideas regarding where they wanted to locate the device. Some didn't want it in their living room, as this was where they watched t.v. Most devices were placed in the kitchen. However, 2 devices were placed upstairs in a spare room, which meant that participants would not always hear an incoming call on the device. In these 2 cases, participants were living with a spouse and felt the device would be best placed in a room that the spouse didn't often use. There were a number of issues raised regarding the call feature and the participant's control over it. Mainly these fell into one of two categories: time and people. There are certain times that participants would not like to be contacted, not only on the Building Bridges device, but also through their home or mobile phone. One participant said she often turns off her mobile phone when she is out to avoid being contacted. Likewise, during their favourite television programs, some participants would not answer their personal phone and some stated they would turn off the device to ensure they were not disturbed: ".. the phone(Building Bridges phone) ringing at the wrong time is a nuisance, same as your own phone would be, and I wish there was a way of staying off it without having to turn the whole thing off. Because if you turn the whole thing off you might forget to turn it on again, you might miss a broadcast or forget, you know. In my mind, that's one of the bad things about it". This idea of liking to contact people but not always wanting to be available to be contacted by others is alluded to by Lindley et al in [13]. The authors talk about older adults delighting in asymmetry whereby they are happy to monitor their young relations but do not want their young relations monitoring them. Conversations with participants following the trial also revealed concerns about having to talk to certain people you might not like. One participant said the following about a neighbour: "Ihave one person whocalls me every morning, you can't get away". There was also a worry that on the device you might start a conversation with someone, but then not be able to 'get away'. This led to the idea of putting a time limit on calls. A twenty minute timer is placed on the screen during calls so all participants can see the remaining time. As mentioned earlier, one participant did not like to make calls as he didn't feel comfortable being the person to make contact: "They rang me all the time, but I admit that Ididn't do the ringing bit. Iwas kind of avoiding thatunless Ireallyhad to, you know". However, he did state that he really liked to receive a call. As such, a potential usage of the system may involve two groups of people, callers and receivers, whereby callers are volunteers who make contact on a regular basis. Of particular interest to us was how participants would react to the group call feature, given that this is one of the benefits of the device over a regular phone. We integrated a number of features into the group call interface to ensure it was easy to use and to try to maintain a certain level of order. As such we added an 'I want to make a comment' button, with the idea that it would substitute for 'putting up one's hand'. When a participant pressed this button their avatar would bounce up and down on other participants' screens. However, in reality if there are many people on a call it is likely that there will be more than one person pressing this button at any one time. As one participant commented, a moderator would be useful: "Very early on we had a full house.. you had 5 people trying to get into a conversation, you had to cut in across each one you know, and l'd say if you were on a short fuse..(annoyed)...I think.. you really wanted a chairperson". We also felt it was important that participants could see who was talking, despite the system not being video-based. We used the concept of speech bubbles on a participant's avatar when they were speaking, but this caused some confusion: "Once I was a bit confused, there were 3 of us and I didn't know who was talking to me half the time, except I knew it was a male voice or a female voice". Another participant noted: "If you could see each other, you'd get an indication of when to stop and when the other person could come in". However, while some participants thought that video 
conferencing would be a good addition, most felt they would only like to try it with close friends and family. As such, evaluating different ways to convey this information is a topic of future work. Overall, while the idea of a group call was well perceived and participants felt it would be very useful for contacting more than one family member at once, there are a number of technical issues that need to be resolved. With regard to messages, the importance of written communication was highlighted by participants. The messaging function was commonly used to let other participants know that someone was going to be away for a couple of days, for example. Group messaging was used by Group 1 to send Christmas wishes to all. Messaging was also used to let people know that someone had tried to make contact with them, as we had not included a function which showed missed calls. This has since been rectified. As mentioned previously, the space provided was considered too short to write anything meaningful. Participants also commented on the importance of correct grammar and spelling: "Well.. sending the messages, if there was a way of going back if you made a mistake, acursor thing or whatever you call it, if you'd spelt something wrong. I don't mean there should be a spell check, I've always been an ok speller. But sometimes when I write a word it looks wrong and I'm dubious about it". Participants typically enjoyed the messaging function. One spoke of how she loved to return to the device and find a new message waiting to be read. Another commented that it gave her more confidence to text on her mobile: "Ah yeah, I do a bit more texting on my mobile now than I used to so it has got me used to it". With regard to the keyboard itself most participants stated they would prefer a qwerty keyboard: "I'm used to a typewriter keyboard and this is different, just a-z going one after the other.. it's not like a typewriter".As such, we plan to have the keyboard as a customisable feature on the device, allowing participants to choose qwerty or abc depending on their preferences.

\subsubsection{Making Friendships -The Importance of Common interests}

During post trial interviews with participants we asked about the nature of conversations on the device, attempting to understand the dynamics of friendship formation in this manner. What emerged was that at the beginning, participants tried to establish a common ground, whether this be through shared experiences, or shared interests: ".. he was talking about something one day and it was something I was interested in too.. It turned out we were both involved in a community of some sort, it was music he was involved in too, choirs and things. I think we had quite a bit in common. Ireally enjoyed our chats". Another participant also mentioned the importance of common interests: "Pointless it is, talking to people you have nothing to do with". In further reference to the notion of building user models for Building Bridges, we may also try to link people with similar interests. For example, a support group for those suffering from a health issue, or for carers. Issues relating to connecting men and women on such a device were also raised. One man commented that when he joined a call after a broadcast there were "just a lot of women gabbing (talking) on".Another female participant felt her husband would not like it if she were contacting other men on the device. A common feeling that emerged was that the system could not take the place of face to face contact, and that at some point during communication with other participants, this would be necessary. One participant suggested a facilitated meeting with all participants might have been useful before beginning the trial. While the idea at this stage was for participants to begin initial communication through the device, it is something to consider for the future.

\section{CONCLUSION AND FUTURE WORK}

This paper has presented details of an in-home evaluation of a touch screen communication device for older adults. Allowing older adults to trial a technology over a period of time helps them to develop informed opinions on the potential benefits of such technology and allows designers and developers to assess real usage patterns, log interactions in real contexts of use and to channel such rich data into a usable end product that older adults are motivated to use and that ultimately enhances their social network. Particularly, we found including error correction facilities is of high importance to increase the perception of the user, providing them with feedback as to whether or not their actions are being recognised by the system. While usability is a critical factor in ensuring such technology is adopted by older adults, it is also necessary to understand their attitudes towards using communication technology. Through our posttrial discussions with participants, it emerged that to be motivated to use a technology on a regular basis, older adults must perceive the technology as a benefit in their current lives. Furthermore, there are certain times when most of our participants would not wish to be contacted. Giving older adults the opportunity to test such technology provided us with invaluable feedback regarding their opinions of communication in later life. All of this has fed into our current design of the device. One of the main factors to consider for future work is how to sustain user motivation to use the system in the long term. Developing models of usage may be one way to tackle this, in addition to introducing the device to already established networks of older adults, such as an ARG. In addition we plan to host facilitated discussions on topics of interest in the tea room and to integrate an information 
and services features, providing users with up to date information regarding services available to them in their area. Finally, following completion of our current trial, which involves a cohort of 20 older adults and approximately 40 of their friends and family members, we will carry out a detailed study to determine to what extent our redesign decisions have had a positive effect on usability. Once we are satisfied that the device is usable and features to encourage usage, we will conduct RCTs with different groups of users, including socially isolated. Acknowledgments. This research was completed as part of a wider programme of research within the TRIL Centre, (Technology Research for Independent Living). The TRIL Centre is a multi-disciplinary research centre, bringing together researchers from UCD, TCD, NUIG \& Intel, funded by Intel, IDA Ireland and GE Healthcare. www.trilcentre.org. We would particularly like to thank our colleagues on the Building Bridges team, especially Joe Wherton, David Prendergast, Brian Lawlor, James Brennan, Vanessa Buckley, Blaithin O'Dea, Simon Roberts, Cormac Sheehan, David Singleton, Claire Somerville, Susan Squires, Maurice ten Koppel, Flip van den Berg and Ciaran Wynne.

\section{REFERENCES}

[1] S. Cohen, L. Underwood, and B. Gottlieb. Social Support Measurement and Intervention: A Guide for Health and Social Scientists. Oxford University Press, 2000. [2] S. Czaja, N. Charness, A. Fisk, C. Hertzog, S. Nair, W. Rogers, and J. Sharit. Factors predicting the use of technology: findings from the center for research and education on aging and technology enhancement (create). Psychol Aging,21(2):333-352, 2006. [3] S. Czaja, J. Guerrier, S. Nair, and T. Landauer. Computer communication as an aid to independence for older adults. Behaviour and Information Technology,12(4):197-207, 1993.

[4] T. Dalsgaard, M. Skov, and B. Thomassen. ekiss: Sharing experiences in families through a picture blog. In British Computer Society Conference on Human Computer Interaction, pages 67-75, 2007. [5] A. Dickinson, A. Newell, M. Smith, and R. Hill. Introducing the internet to the over 60s: developing an email system for older novice computer users. Interacting with Computers, 17:621-642, 2005.

[6] R. Findlay. Interventions to reduce social isolation amongst older people: where is the evidence? Ageing and Society,23(5):647-658, 2003.

[7] D. Gardner and E. Helmes. Locus of control and self-directed learning as predictors of well-being in the elderly. Aust Psychol,34(2), 1999.

[8] J. Haiko, M. Isomursu, T. Matinmikko, A. Wallin, H. Ailisto, and T. Huomo. Touch based user interface for elderly users. In Mobile $\mathrm{HCl}$ '07, pages 289-296, 2007.
[9] D. Hawthorn. Possible implications of aging for interface designers. Interacting with Computers, 12:507-528, 2000.

[10] B. Holt and R. Morrell. Guidelines for website design for older adults: the ultimate influence of cognitive factors. In Older Adults, Health Information and the World Wide Web, pages 109-132, 2002.

[11] A. Holzinger. Finger instead of mouse: Touch screens as a means of enhancing universal access. In Theoretical Perspectives, Practise and Experience, pages 387-397, 2003.

[12] C. Jones, C. Winegarden, and W. Rogers. Supporting healthy ageing with new technologies. ACM Interactions, 16(4):48-51,2009.

[13] S. Lindley, R. Harper, and A. Sellen. Designing for elders: exploring the complexity of relationships in later life. In British Computer Society Conference on Human Computer Interaction, pages 77-86, 2008.

[14] S. Lindley, R. Harper, and A. Sellen. Desiring to be in touch in a changing communications landscape: Attitudes of older adults. In $\mathrm{CHI}$ '09, pages 1693-1702, 2009.

[15] A.-S. Melenhorst, W. Rogers, and E. Caylor. The use of communication technologies by older adults: exploring the benefits from the user's perspective. In Human Factors and Ergonomics Society 45th Annual Meeting,2001.

[16] F. S. Salces, M. Baskett, D. Llewelyn-Jones, and D. England. Ambient interfaces for elderly people at home. In Ambient Intelligence in Everyday Life, pages 256-284, 2006.

[17] T. Sokoler and M. S. Svensson. Presence remote: Embracing ambiguity in the design of social tv for senior citizens. In Changing Television Environments 6th European Conference EUROITV, pages 158-162, 2008.

[18] J. Wherton and D. Prendergast. The building bridges project: involving older adults in the design of a communication technology to support peer-topeer social engagement. In $\mathrm{HCl}$ and Usability for E-Inclusion, pages 111-134, 2009.

[19] H. White, E. McConnell, E. Clipp, and L. Branch. A randomized controlled trial of the psychosocial impact of providing internet training and access to older adults. Ageing and Mental Health,6:213-221, 2002.

[20] M. Zajicek. Interface design for older adults. In Workshop on Universal Accessibility of Ubiquitous Computing, pages 60-65, 2001.

[21] M. Zajicek. Aspects of hci research for older people. Universal Access in the Information Society, 5(3):279-286, 2006. 